\title{
FORTALECIMIENTO DEL PENSAMIENTO VARIACIONAL A TRAVÉS DE LA GAMIFICACIÓN Y LA TEORÍA DE LA OBJETIVACIÓN
}

\section{STRENGTHENING VARIATIONAL THINKING THROUGH GAMIFICATION AND OBJECTIFICATION THEORY}

\section{Ingrid Milena Parra-Arenales}

\section{Wilson Andrés Martínez-Delgado}

\section{Élgar Gualdrón-Pinto}

Universidad Autónoma de Bucaramanga

Universidad de Pamplona

\section{RESUMEN}

En el este artículo se presentan los resultados de una investigación cualitativa, realizada con estudiantes de octavo grado de la básica secundaria (13 a 16 años), y que buscaba fortalecer el pensamiento variacional de los estudiantes a través de una estrategia didáctica basada en la gamificación como elemento

Ingeniera Electrónica, Estudiante de Maestría en Educación-Universidad Autónoma de Bucaramanga. Iparra308@unab.edu.co; ORCID: https://orcid.org/0000-00017081-6062

Especialista en Telecomunicaciones, Estudiante de Maestría en Educación-Universidad Autónoma de Bucaramanga.wmartinez223@unab.edu.co; ORCID: https://orcid. org/0000-0002-4457-0772

Doctor en Didáctica de las Matemáticas; Profesor Titular en la Universidad de Pamplona; director del Grupo de Investigación EDUMATEST de la Universidad de Pamplona. egualdron@unipamplona.edu.co; ORCID: https://orcid. org/0000-0002-4081-2092 motivacional y adaptando las actividades a desarrollar según lo planteado en la teoría de la objetivación propuesta por Luis Radford. El desarrollo contempló tres etapas: el diagnóstico, a partir del cual se identificó el nivel inicial del pensamiento variacional en los estudiantes; la implementación de la estrategia didáctica, que integraba las temáticas a desarrollar el primer periodo académico según lo establecido en plan de área institucional; y la prueba de cierre, aplicada con el fin de valorar el impacto de la estrategia didáctica. El análisis de los resultados a través de las categorías de análisis refleja que tanto el factor motivacional como el desarrollo del pensamiento variacional fueron afectados favorablemente tras la implementación de la estrategia didáctica.

PALABRAS CLAVE: Pensamiento variacional, gamificación, teoría de la objetivación. 


\section{ABSTRACT}

This article presents the results of a qualitative research, developed with eighth grade high school students (13 to 16 years old), which sought to strengthen the variational thinking of students through a didactic strategy based on gamification as a motivational element and adapting the activities to be developed according to the theory of objectification proposed by Luis Radford. The development included three stages: the diagnosis, from which the initial level of variational thinking in students was identified; the implementation of the didactic strategy, which integrated the topics to be developed in the first period as established in the institutional area plan; and the closing test, applied in order to assess the impact of the didactic strategy. The analysis of the results through the categories of analysis reflects that both the motivational factor and the development of variational thinking were favorably affected after the implementation of

\section{KEYWORDS}

Variational thinking, gamification, objectification theory.

\section{INTRODUCCIÓN}

A través de los años, el aprendizaje de la matemática escolar ha sido objeto de diversos estudios a nivel mundial, con el propósito de precisar las dificultades que enfrentan los estudiantes en este proceso y que además permita el desarrollo de estrategias eficientes que conlleven al logro de dicho aprendizaje (Kieran y Filloy, 1989). Los estudios han reflejado diferentes problemáticas que generan resultados desfavorables en el área, situación que se incrementa al iniciar el estudio formal del álgebra (Martínez-López y Gualdrón-Pinto, 2018; Ordóñez-Ortega, Gualdrón-Pinto y Amaya-Franky, 2019), lo que se evidencia en un mayor fracaso escolar (Gavilán, 2011). Si bien

se ha concluido que las dificultades se generan debido a múltiples factores relacionados tanto con el entorno socio-cultural, como con situaciones propias del proceso de enseñanza aprendizaje, la presente investigación se centra específicamente en el estudio del desarrollo del pensamiento variacional.

\section{FUNDAMENTACIÓN TEÓRICA}

El pensamiento variacional hace referencia a una forma de pensamiento dinámico que busca la producción mental de sistemas, en los cuales sus variables se relacionan en forma tal que covaríen de acuerdo a los patrones de covariación de cantidades de la misma o distintas magnitudes relacionadas en sucesos extraídos de la realidad (Vasco, 2003), y está relacionado "con la percepción, la identificación y la caracterización de la variación y el cambio en diferentes contextos, así como con su descripción, modelación y representación en distintos sistemas o registros simbólicos, ya sean verbales, icónicos, gráficos o algebraicos" (MEN, 2006, p. 66).

Este pensamiento se desarrolla a lo largo de la educación básica y media, enlazado con los otros cuatro tipos de pensamiento (numérico, espacial, métrico y aleatorio), tal como se ilustra en la figura 1, a fin de fortalecer en los estudiantes el uso de conceptos y procedimientos relacionados con los diferentes sistemas, para consolidarlos como herramienta en la resolución de problemas matemáticos, de las ciencias sociales, naturales y de la vida cotidiana, que demanden el estudio de la variación, el cambio y la modelación de procesos (MEN, 2006). 
Figura 1. Relación del pensamiento variacional con los demás pensamientos.

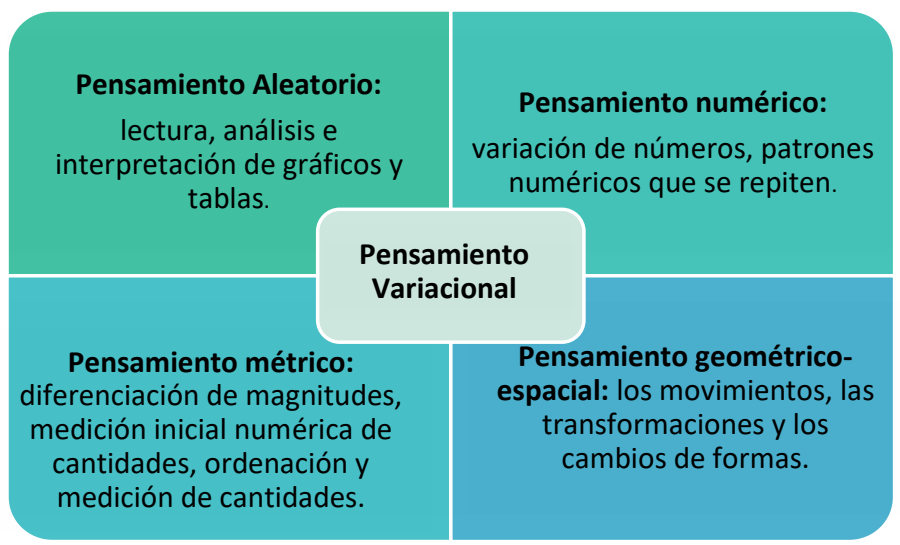

Nota. Fuente: MEN (2006).

Es preciso indicar que el desarrollo del pensamiento variacional no se limita solo a los sistemas algebraicos, sino que se debe iniciar desde los primeros años, implementando actividades que propicien procesos básicos de generalización, variación y argumentación (Martínez-López y Gualdrón-Pinto, 2018). Por ende, un estudiante que ingrese al grado octavo (13 a 16 años) debería estar en capacidad de identificar en una situación lo que varía y lo que permanece constante; expresar en lenguaje común o simbólicamente generalidades relacionadas con los conceptos aritméticos y determinar los patrones en secuencias gráficas, geométricas o numéricas sencillas, así como también poder argumentar las decisiones tomadas usando propiedades matemáticas.

\section{TEORÍA DE LA OBJETIVACIÓN}

La teoría de la objetivación es una teoría sociocultural contemporánea inspirada en el materialismo dialéctico y la escuela vygotskiana, sobre la enseñanza-aprendizaje de las matemáticas que "plantea el objetivo de la educación matemática como un esfuerzo político, social, histórico y cultural cuyo fin es la creación de individuos éticos y reflexivos que se posicionan de manera crítica en prácticas matemáticas constituidas histórica y culturalmente" (Radford, 2014a, p. 135-136).

Esta teoría tiene como eje central el concepto de actividad o labor y propone el proceso de enseñanza-aprendizaje como una actividad unificada, en la cual se producen saberes y subjetividades a partir de la labor conjunta, entendida esta como un sistema de interacción dinámico entre docente y estudiantes, que tiene como objetivo encontrar sistemas de pensamiento, que permiten al sujeto alcanzar una realización propia y social (Radford, 2014b). El concepto de actividad se define como un sistema en movimiento que se estructura en relación a su intención pedagógica en la clase, mediante un proceso de objeto- meta- tarea. La actividad inicia teniendo un objeto claro que se funda en el proyecto didáctico, posteriormente para que la actividad logre el objeto se establecen una o varias metas que serán alcanzadas a partir del desarrollo de tareas específicas que se sugiere tengan un grado de dificultad conceptual creciente (Radford, 2017).

Así mismo, para llevar a cabo una actividad, se suelen dividir la totalidad de los estudiantes en grupos de tres o cuatro, y posteriormente se establecen los siguientes momentos: 
- Presentación de la actividad

- Trabajo en grupos

- Visita del docente para interacción y retroalimentación

- Socialización general con aportes entre grupos

- Retorno al trabajo en grupos (Opcional)

La actividad se desarrolla dentro de un espacio y tiempo previamente establecido y responde a una planeación didáctica, no obstante, su dinámica no se puede determinar con certeza por anticipado, toda vez que no es un proceso mecánico ni determinístico, pues influyen diversos factores (Radford, 2017).

\section{GAMIFICACIÓN}

La gamificación es un término que se ha utilizado en los últimos años y al que se le han dado diversas definiciones e interpretaciones dependiendo del contexto de aplicación. Para la presente investigación se define como: un proceso que influye en la motivación y el comportamiento de los usuarios a partir del uso del pensamiento y las mecánicas del juego, para involucrarlos y resolver problemas (Zichermann y Cunningham, 2011).

Los juegos influyen en el factor motivacional ya que dan seguridad, proporcionan experiencias, establecen límites y promueven la autonomía dentro de un entorno en el que es permitido equivocarse, lo que reduce la frustración ante el fracaso, ya que existe la posibilidad de reiniciar y ganar. Gamificar no se puede limitar a la asignación de puntos, insignias y recompensas, por el contrario, debe buscar despertar los sentimientos de emoción y satisfacción que caracterizan los juegos (Kapp, 2012).

A fin de tener una guía estructurada para llevar a cabo el proceso de gamificación el Observatorio de Innovación Educativa del Tecnológico de Monterrey (2016) propone definir nueve elementos:

1. Objetivo: definir el o los objetivos de aprendizaje.

2. Perfil de los Jugadores: Los usuarios de gamificación se pueden clasificar según motivaciones e intereses.

3. Comportamientos esperados: Se realiza una lista en la que se describa las acciones esperadas de los estudiantes y las preexistentes que se desean mejorar

4. Componentes: Definir los elementos a incorporar como niveles, regalos, avatares, puntos, entre otros.

5. Mecánicas: Definir las actividades a implementar como retos, suerte, competencias. Según los objetivos y entorno

6. Dinámicas: En este paso se determinan las reglas de juego, la narrativa, las emociones.

7. Gestión: Se establecen acciones y recursos para dar seguimiento al proceso por parte del docente y de los estudiantes.

8. Riesgos potenciales: Analizar los posibles riesgos que puedan existir, ya sean accidentales $o$ actitudinales.

9. Estéticas: Finalmente se eligen los elementos que darán identidad y carácter, como: selección de colores, entornos, avatares.

A fin de conseguir una experiencia gamificada exitosa, se requiere un acompañamiento, una retroalimentación constante y una disposición de cambio, en pro de mejorar el proceso y evitar el riesgo de la monotonía, la deserción y el fracaso de la estrategia. El docente debe asumir un rol activo dentro del proceso, generando espacios y 
ambientes participativos, en los cuales se haga efectiva, para los estudiantes, la posibilidad de equivocarse, haciendo de esta una experiencia de aprendizaje que permita superar la frustración a través de la incorporación de actividades innovadoras y atractivas que mantengan su interés.

\section{ESTRATEGIA DIDÁCTICA}

La estrategia didáctica hace referencia al conjunto de acciones que se realizan con el fin de alcanzar una meta propuesta e impulsar procesos de aprendizaje, por tal motivo, es indispensable plantear un objetivo hacia el cual se orienten las técnicas utilizadas y las actividades diseñadas. Teniendo en cuenta las características propias de los alumnos, la estrategia debe ser flexible y ofrecer la opción de cambio en dirección al cumplimiento de las metas propuestas (Velasco y Mosquera, 2007).

\section{METODOLOGÍA}

220

El trabajo responde a una investigación cualitativa toda vez que según Bonilla-Castro y Rodríguez (1997): "El proceso de investigación cualitativa explora de manera sistemática los conocimientos y valores que comparten los individuos en un determinado contexto espacial y temporal" (p.86). Ya que el trabajo busca modificar la dinámica establecida dentro de las clases con el fin de fortalecer el desarrollo del pensamiento variacional, se aduce a la investigación acción como el tipo de investigación oportuno, en tanto que esta se enmarca dentro de un proceso continuo de acción y reflexión entre procesos y contextos específicos (Hernández, Callado, y Baptista, 2010). La investigación se estructura a partir de una serie de pasos (planificación, acción, observación y reflexión) según el planteamiento realizado por Carr y Kemmis (1988) que se relacionan en forma cíclica denominados etapas:
Planificación: se realizó una revisión de literatura que sirvió como referente para establecer cuáles son los indicadores que permiten analizar el desarrollo del pensamiento variacional y así establecer las categorías de análisis. Conjuntamente, llevó a definir los teóricos y constructos a partir de los cuales se desarrolló la propuesta pedagógica, estableciendo una relación entre contenidos, entorno y actividades que propicien un ambiente favorable para el proceso de aprendizaje.

Acción: se realizó la intervención a los estudiantes aplicando la estrategia didáctica diseñada previamente, que integra características propias de gamificación en búsqueda de aumentar la motivación en lo referente al compromiso con su proceso de aprendizaje, y cuyas actividades responden a la secuencia basada en la teoría de la objetivación que buscan favorecer el aprendizaje desde la interacción con sus compañeros.

Observación: esta fase se realiza en simultáneo con la fase de acción y en forma permanente, la finalidad es observar durante el desarrollo de las actividades las interacciones y aportes realizados por los estudiantes llevando un registro con ayuda del diario de campo donde se consigne la información relacionada con la parte actitudinal, motivacional y procedimental.

Reflexión: la fase de reflexión se dividió en dos partes, la primera es la que se realiza finalizado cada encuentro dentro de las primeras sesiones en las que se evalúa principalmente la eficiencia de la interacción y el avance de las actividades ya que la metodología de trabajo es novedosa dentro de la virtualidad. La segunda parte consiste en una reflexión más profunda sobre los resultados actitudinales y de conocimiento frente a la problemática planteada, con el fin de adecuar la propuesta en pro de lograr el objetivo propuesto. 


\section{CATEGORÍAS DE ANÁLISIS}

En la tabla 1 se presentan las categorías de análisis seleccionadas en base a la revisión de literatura y el propósito de la investigación.

Tabla 1. Categorías de análisis

\begin{tabular}{|c|c|c|}
\hline Categorías & Subcategorías & Indicadores \\
\hline \multirow{10}{*}{$\begin{array}{l}\text { Pensamiento } \\
\text { variacional }\end{array}$} & \multirow{3}{*}{$\begin{array}{l}\text { 1. Generalización de las } \\
\text { propiedades aritméticas }\end{array}$} & $\begin{array}{l}\text { 1.1. Razona y propone explicaciones sobre las propiedades de la } \\
\text { igualdad en tareas de relación entre cantidades. }\end{array}$ \\
\hline & & $\begin{array}{l}\text { 1.2. Expresa generalizaciones y construye argumentos } \\
\text { coherentes sobre las propiedades de las operaciones. }\end{array}$ \\
\hline & & $\begin{array}{l}\text { 1.3. Expresa generalizaciones sobre las propiedades de los } \\
\text { números mediante el lenguaje natural, diagramas o símbolos } \\
\text { algebraicos. }\end{array}$ \\
\hline & \multirow{3}{*}{$\begin{array}{l}\text { 2. Generalización y } \\
\text { razonamiento sobre la } \\
\text { covariación }\end{array}$} & $\begin{array}{l}\text { 2.1. Analiza de qué forma cambia, aumenta o disminuye } \\
\text { la forma o el valor en una secuencia o sucesión de figuras, } \\
\text { números o letras. }\end{array}$ \\
\hline & & $\begin{array}{l}\text { 2.2. Realiza conjeturas sobre la forma o el valor del siguiente } \\
\text { término de la secuencia expresando los siguientes términos por } \\
\text { escrito o a través de dibujos u otras representaciones. }\end{array}$ \\
\hline & & $\begin{array}{l}\text { 2.3. Formula un procedimiento algoritmo o fórmula que permita } \\
\text { reproducir el mismo patrón, calcular el enésimo término. }\end{array}$ \\
\hline & \multirow{4}{*}{$\begin{array}{l}\text { 3. Capacidades } \\
\text { relacionadas con la } \\
\text { sintaxis algebraica }\end{array}$} & 3.1. Halla el valor de la incógnita en ecuaciones \\
\hline & & $\begin{array}{l}\text { con la contengan en uno o en ambos lados del signo igual a } \\
\text { partir de la estrategia de preferencia. }\end{array}$ \\
\hline & & $\begin{array}{l}\text { 3.2. Formula relaciones utilizando símbolos algebraicos } \\
\text { mediante tareas que requieren la traslación de expresiones } \\
\text { verbales/escritas a ecuaciones, expresiones y fórmulas } \\
\text { algebraicas o viceversa. }\end{array}$ \\
\hline & & $\begin{array}{l}\text { 3.3. Realiza operaciones con símbolos algebraicos y simplifica } \\
\text { expresiones algebraicas. }\end{array}$ \\
\hline \multirow[t]{3}{*}{ Motivación } & \multirow{3}{*}{$\begin{array}{l}\text { Actitud frente a las } \\
\text { clases }\end{array}$} & Asiste puntualmente a todos los encuentros virtuales. \\
\hline & & $\begin{array}{l}\text { Participa activamente en todas las sesiones realizando aportes } \\
\text { respetuosos y pertinentes. }\end{array}$ \\
\hline & & $\begin{array}{l}\text { Presenta las actividades en forma oportuna y cumpliendo con } \\
\text { los criterios establecidos. }\end{array}$ \\
\hline
\end{tabular}

Nota. Elaboración propia con datos tomados de Chrysostomou y Christou (2019). 
En relación al pensamiento variacional, las subcategorías y los indicadores fueron establecidos con base en el trabajo realizado por Chrysostomou y Christou (2019), y a los estándares básicos de competencias en matemáticas del MEN (2006). En lo referente a la motivación, se analizó a partir de las actitudes que se evidenciaron durante el desarrollo de las clases, especialmente frente a la situación de virtualidad en la cual se generó un proceso de flexibilidad en respuesta a las limitaciones de acceso de algunos estudiantes.

\section{ESTRATEGIA DIDÁCTICA}

Con el fin de fortalecer el desarrollo del pensamiento variacional en los estudiantes de octavo grado, se diseña la estrategia didáctica "El bosque de los números" basada en gamificación que integra elementos de la teoría de la objetivación en lo referente a la dinámica de las actividades. La propuesta fue implementada durante el primer periodo académico del año 2021 a través de un entorno virtual, atendiendo a la modalidad de estudio del momento, dada la situación de pandemia generada por el COVID19, y responde a las siguientes características de diseño:

El bosque de los números: Los guardianes del bosque de los números buscan sembrar y preservar las cinco especies de árboles que más oxigeno aportan, para ayudar a combatir la problemática ambiental que ha surgido ante el aumento de la industria y el consumismo desmedido, lo que conlleva a que cada día se destruyen más bosques, los cuales son fuente generadora de oxígeno.
Objetivos: Inicialmente, se establecen los objetivos de aprendizaje que se buscan alcanzar al implementar la estrategia didáctica, los cuales van acorde con los establecidos en el plan de área.

Perfil de los Jugadores: Estudiantes entre 13 y 16 años, en constante interacción con las herramientas tecnológicas, especialmente las redes sociales y una creciente inmersión en los videojuegos.

Comportamientos esperados: Se espera que al implementar la estrategia basada en gamificación los estudiantes aumenten su interés por ser partícipes de la clase, no solo como asistentes, sino como parte activa de su proceso educativo.

Componentes: Los estudiantes son representados por avatares llamados guardianes del bosque que deben sembrar las cinco especies de árboles que más oxígeno proporcionan. La tabla 2 ilustra como la siembra se genera a partir del desarrollo de diferentes actividades, y la puntuación que reciben los estudiantes, quienes suben de nivel al conseguir la puntación requerida, hasta llegar al máximo nivel con 600 puntos. 
Tabla 2. Generación de siembra a partir de actividades realizadas

\begin{tabular}{|l|c|c|c|}
\hline \multirow{2}{*}{$\begin{array}{l}\text { Especie de Árbol } \\
\text { sembrado }\end{array}$} & \multicolumn{3}{|c|}{ NIVEL } \\
\cline { 2 - 4 } & 1 & 2 & 3 \\
\hline $\begin{array}{l}\text { Pino carrasco } \\
\begin{array}{l}\text { (Actividades en } \\
\text { clase) }\end{array}\end{array}$ & 30 & 35 & 600 \\
\hline $\begin{array}{l}\text { Árbol kiri } \\
\text { (Actividades en } \\
\text { casa) }\end{array}$ & 25 & 30 & 35 \\
\hline Pino Piñonero & 20 & 25 & 30 \\
(Participación) & 10 & 15 & 20 \\
\hline $\begin{array}{l}\text { Alcornoque } \\
\text { Mediterráneo }\end{array}$ & 20 & 25 \\
(puntualidad) & 15 & & \\
\hline $\begin{array}{l}\text { Jacaranda } \\
\text { (Actividades } \\
\text { adicionales) }\end{array}$ & & & \\
\hline
\end{tabular}

Nota. Fuente: elaboración propia.

El progreso de los estudiantes es informado en cada encuentro virtual en el cual se presenta la tabla de posiciones exaltando los 10 guardianes que más árboles han sembrado.

Mecánicas: Los estudiantes deben desarrollar las actividades planteadas en clase siguiendo los momentos propuestos por la teoría de la objetivación, iniciando cada encuentro virtual en una sala general en la que se comparte la guía de trabajo e indicaciones generales; posteriormente en grupos conformados por cinco estudiantes trabajan en salas individuales, a las cuales el docente ingresa en diferentes momentos; en un tercer momento, los estudiantes vuelven a la sala general para realizar una discusión entre grupos $\mathrm{y}$, finalmente, cada grupo se reúne para realizar ajustes si lo consideran necesario y concertar el producto de entrega final.
Las actividades en casa son desarrolladas en forma individual y valoradas dependiendo del tiempo de entrega, la presentación y el contenido, estas son revisadas y los estudiantes tienen la opción de hacer las correcciones que se requieran. Por otro lado, las actividades adicionales son de desarrollo voluntario $y$ se asignan constantemente, consisten en ejercicios que se deben desarrollar en línea, de retroalimentación automática y con un grado de dificultad creciente.

Dinámicas: El desarrollo de todas las actividades busca despertar en los estudiantes una conciencia de conservación por el medio ambiente, la presentación general de los avances se hace enfatizando cuántos árboles ha plantado y su impacto positivo, en cuanto al progreso individual este puede ser consultado en cualquier momento por los estudiantes en la plataforma.

Gestión: La plataforma ClassDojo fue seleccionada para que los estudiantes puedan dar seguimiento a su progreso, personalizar su avatar, mantenerse al tanto sobre las actividades adicionales e interactuar con los compañeros y el docente. La plataforma seleccionada es de acceso libre, presenta un entorno amigable y da la opción de ingreso desde el celular ya que cuenta con aplicación móvil.

Riesgos potenciales: Según las características de la población y dada la condición de virtualidad uno de los riesgos que se afronta es que los estudiantes deserten bien sea por la resistencia al cambio o por las condiciones económicas que afrontan las familias, lo que demanda un seguimiento permanente a la dinámica de clase.

Estéticas: Desde la presentación del bosque de los números se involucran visualmente los 5 árboles incluidos en la narrativa y los avatares de la plataforma ClassDojo, por lo que los colores predominantes en las presentaciones 


\section{RESULTADOS}

\section{Prueba diagnóstica}

En relación a las categorías de análisis establecidas para el pensamiento variacional los resultados observados en la prueba diagnóstica reflejaron que:

Los estudiantes no tienen claridad conceptual ni procedimental sobre las propiedades de las operaciones, a pesar de ser un tema aborda desde la básica primaria, resultado ya manifestado por Ordóñez-Ortega, GualdrónPinto y Amaya-Franky (2019). En ejercicios que demandan comprender la igualdad frente a una variación en la sintaxis de la propiedades distributiva y conmutativa se evidencia que no existe apropiación, pues recurren a realizar las operaciones numéricas para comprobar los postulados. Al momento de presentar justificaciones sobre la aplicación de la propiedad conmutativa recurren a enunciar la propiedad asociativa. Comportamiento similar se observa con las propiedades de los números, donde los estudiantes conocen las características de los números como es el caso de los pares e impares, pero no logran establecer generalidades al operarlos y siempre deben recurrir a ejemplos numéricos. En cuanto al concepto de igualdad, se observa mayor dominio en ejercicios que involucran representaciones gráficas $y$ expresiones literales sencillas.

Los estudiantes identifican el cambio de formas o valor en secuencias o sucesiones numéricas y figurales, consiguen predecir la forma o valor del siguiente término o de una posición específica, a través de procesos gráficos o de conteo, pero sin llegar a establecer una fórmula que permita hallar el enésimo término. Algunas expresiones planteadas por los estudiantes para conseguir una generalización se centran en lo que cambia sin tener en cuenta la presencia de constantes.
Los estudiantes hallan el valor de la incógnita en ecuación sencillas, que no requieren algoritmos para determina su valor. Realizan remplazos asignando valores numéricos a situaciones que presentan en forma verbal o escrita modelos de cambio, no obstante, no consiguen trasladar la situación a lenguaje algebraico.

La prueba diagnóstica proporcionó información valiosa que permitió organizar y priorizar las temáticas a abordar, reflejando la necesidad de retomar el estudio de conceptos y procedimientos aritméticos como las propiedades de los números y las operaciones, así como los algoritmos para resolver operaciones básicas, que si bien fueron abordados en grados anteriores, no fueron apropiados por los estudiantes llegando a proyectar dichas falencias o dificultades al iniciar el estudio formal del álgebra. Lo anterior está en concordancia con lo sugerido por Gavilán (2011) al afirmar que "Muchos estudiantes manifiestan sentimientos de tensión y miedo, que pueden estar asociados al desfase existente entre lo que realmente pueden hacer y lo que se les pide que hagan" (p.98).

\section{Estrategia didáctica}

El análisis de la estrategia se presenta en tres partes, que corresponden a los tres ciclos completos, según lo establecido en la metodología de investigación acción y en los cuales se abordaron las temáticas establecidas para el primer periodo:

\section{Primer ciclo}

Las actividades desarrolladas en este ciclo, en las cuales se buscaba a partir de problemas contextualizados que los estudiantes afianzaran la aplicación de las propiedades de los números e identificaran las propiedades de las operaciones, propician un avance en relación a la subcategoría de generalización de las propiedades aritméticas, no obstante, el progreso no es lo suficientemente significativo 
para alcanzar la apropiación esperada con relación al nivel académico, por lo que es necesario implementar más actividades que refuercen estos pre saberes a lo largo de la estrategia.

En cuanto a la subcategoría de generalización y razonamiento sobre la covariación, se evidencia que los estudiantes progresan en la comprensión de secuencias y predicción literal de patrones al identificar y caracterizar una secuencia numérica.

En el primer ciclo se observa una evolución en cuanto los aspectos de trabajo en grupo, en lo que refiere a la participación. Desde el inicio se evidenció que los estudiantes tenían mayor libertad para expresarse en grupos reducidos, no obstante, limitaban sus aportes relacionados a las actividades o sus intervenciones iban acompañadas de una frase ("es una bobada") o expresión gestual (como el movimiento de la cabeza de un lado a otro) que indicaba desaprobación al considerar que sus comentarios podrían afectar la nota. Este tipo de comportamiento se observó cada vez menos, pues, en la dinámica de grupos, los compañeros no realizaban críticas, y en los que había mayor confianza entre compañeros compartían con bromas o recordando experiencias compartidas. Por otra parte, cuando el docente hacía acompañamiento, los alentaba a que expresaran sus ideas y los guiaba a partir de una discusión y no de una aprobación o reprobación directa.

\section{Segundo ciclo}

En este ciclo se desarrollaron las actividades relacionadas con las expresiones algebraicas y la transformación del lenguaje común a lenguaje algebraico y viceversa, temáticas que permiten observar las capacidades relacionadas con la sintaxis algebraica (subcategoría).

Las interacciones de los estudiantes reflejan la apropiación de conceptos algebraicos y el avance en la comprensión del concepto de variable, al asimilar que no se debe asignar un valor numérico al azar para poder formular relaciones y operaciones, además se observa que los estudiantes, después de realizar el proceso planteado por la teoría de la objetivación sobre el desarrollo de actividades: trabajo en grupos reducidos, intercambio entre grupos, discusión del grupo en pleno y retorno al grupo inicial para finalizar la actividad (Radford, 2017), plantean soluciones que reflejan un avance en su concepción inicial.

Otro aspecto a mencionar es la necesidad de trabajar la subcategoría de generalización de las propiedades aritméticas, puesto que los estudiantes continúan presentando dificultades para identificar y argumentar las propiedades de las operaciones, específicamente la propiedad conmutativa de la suma y la multiplicación en expresiones algebraicas pues refieren que:

$D+6 \neq 6+D \quad, y, \quad(2)(D) \neq(D)(2)$

\section{Tercer ciclo}

Las temáticas abordadas estaban relacionadas con operaciones de suma, resta y multiplicación con expresiones algebraicas, también se debían plantear ecuaciones con una variable, asignar un valor numérico a la variable para solucionar una situación específica y aplicar fórmulas geométricas para hallar el área de cuadriláteros. Una de las actividades consistía en determinar las dimensiones (largo y ancho) de cada uno de los espacios de la casa para, posteriormente, calcular su área. Para determinar la expresión que representaba las dimensiones de algunos espacios se debían aplicar operaciones de suma o resta, y comprender la relación de simetría al ser un espacio rectangular.

En lo referente al desarrollo de las actividades en clase, estas permiten evidenciar que persisten falencias en los procesos aritméticos relacionados con las operaciones básicas, 
siendo más evidentes cuando se operan fracciones, donde falta claridad en los algoritmos que se deben emplear al resolver cada operación: algunos estudiantes suman y restan fracciones heterogéneas en forma lineal, es decir operan los numeradores entre si y este proceso lo replican con los denominadores. Otro error repetitivo está relacionado con la confusión entre la potenciación y la multiplicación. No obstante, en las actividades entregadas y en la interacción con los estudiantes, es posible observar que realizan proceso de generalización al aplicar las propiedades en situaciones en las cuales no se opera con variables.

En cuanto a las capacidades relacionadas con la sintaxis algebraica (subcategoría) se evidencia que: al desarrollar tareas que requieren hallar el valor de la incógnita en ecuaciones que la contengan en un lado del signo igual, los estudiantes realizan el proceso correctamente, pero la respuesta queda sujeta al desarrollo aritmético; cuando se requiere formular relaciones utilizando símbolos algebraicos en tareas que demandan la traslación de expresiones escritas a expresiones algebraicas, realizan con fluidez los planteamientos en forma verbal y escrita pero se evidencia dificultad al concretar la formula; finalmente al realizar operaciones con símbolos algebraicos y simplificar expresiones algebraicas, las operaciones de suma y resta son desarrollados con facilidad, sin embargo en la multiplicación y la división algunos estudiantes presentan dificultades. Lo que sugiere que las dificultades se encuentran en el proceso aritmético y no en el de generalización.

Por otro lado, la posibilidad de ingresar a la plataforma y conocer su progreso personal de forma permanente generó en los estudiantes una actitud de responsabilidad frente a sus obligaciones, estableciendo comunicación por los diferentes medios indagando sobre actividades y dudas sobre algunos temas.

En cuanto al reconocimiento público que se realizaba exponiendo la tabla de posiciones al inicio de cada sesión, se distinguieron dos momentos, inicialmente se evidenciaba que, tras el conocimiento de las posiciones ocupadas, en las reuniones en grupo los estudiantes indagaban por el lugar de sus compañeros, y en un segundo momento, esta dinámica cambió, ya había más una preocupación personal por superar su puntuación que por sus compañeros.

Finalmente, la característica de generar una retroalimentación constante y ofrecer a los estudiantes la posibilidad de comprender cuál es la falla y corregir el error, género en ellos una confianza que se reflejaba en las interacciones con la docente al exponer sus dudas y el realizar planteamientos a pesar de no tener una certeza total.

\section{Prueba de cierre}

La prueba de cierre permite evidenciar que existió una evolución generalizada en el pensamiento variacional al analizar cada una de las subcategorías que lo componen. Lo que se evidencia en la figura 2 donde se expone el comparativo de respuestas correctas en la prueba diagnóstica y la de cierre por estudiante. 
Figura 2. Comparativo respuestas correctas por pruebas.

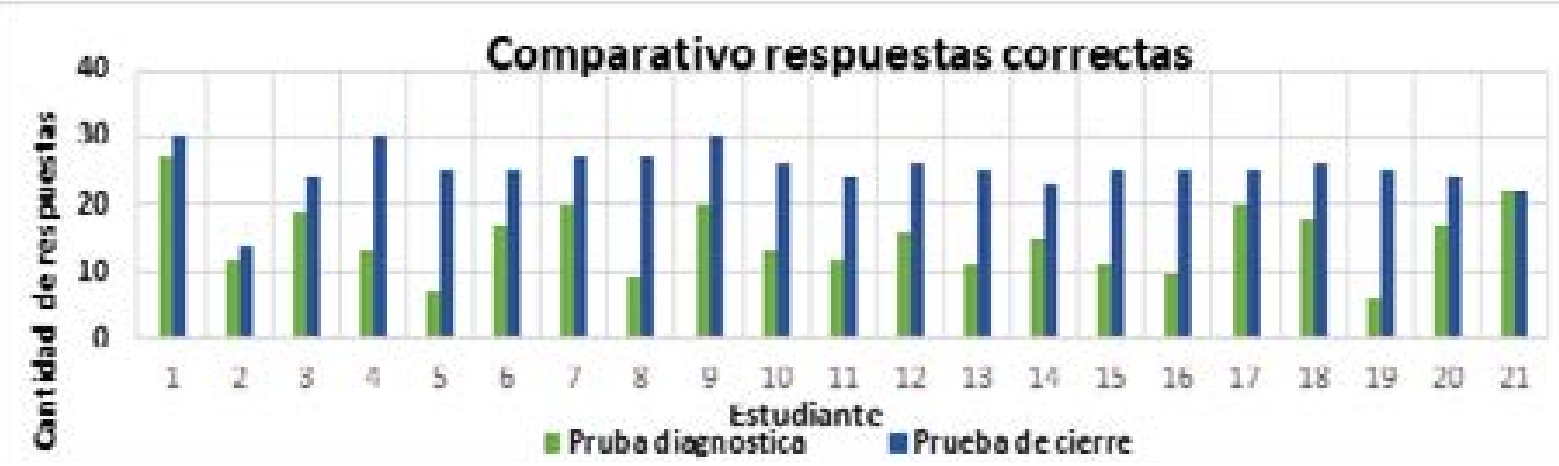

Nota. La figura presenta la cantidad de respuestas correctas obtenidas por cada estudiante en la prueba diagnóstica y la prueba de cierre.

\section{CONCLUSIONES}

Tras implementar la estrategia didáctica se evidencia un fortalecimiento en el pensamiento variacional de los estudiantes en relación a las categorías de análisis establecidas.

Los estudiantes logran plantear generalizaciones de recurrir al uso de ejemplos numéricos y realizan los procedimientos adecuadamente, contrario a lo sugerido por Ordóñez-Ortega, Gualdrón-Pinto y Amaya-Franky (2019), pero persisten dificultades al explicarlas y argumentar su aplicación. Igualmente se evidencia la apropiación del concepto de igualdad en tareas que requieren establecer relaciones entre números, variables y figuras; en cuanto a las propiedades de los números reflejan confusión al momento de aplicar relaciones de orden.

Los estudiantes identifican la variación en secuencias y sucesiones de figuras, números y letras cada vez más complejas, hallan adecuadamente el valor del siguiente término y de los términos para posiciones específicas y plantean el patrón de cambio en forma verbal o escrita, pero no consiguen plantear el modelo a través de expresiones algebraicas.

Los estudiantes hallan correctamente el valor de la incógnita en ecuaciones que la contienen a un lado del signo igual cuando las operaciones se realizan con números enteros; al realizar operaciones con fraccionarios se evidencian dificultades relacionadas directamente con el desarrollo aritmético.

Los estudiantes transforman expresiones algebraicas a lenguaje común y en la mayoría del caso logran realizar el proceso inverso utilizando símbolos algebraicos. En relación a este aspecto se observa que la falta de apropiación y uso adecuado del lenguaje matemático influye negativamente al momento de realizar la transición en los dos sentidos. Por otro lado, la interacción entre compañeros aporta a la apropiación de términos matemáticos adecuados en algunos estudiantes.

Los estudiantes aplican los algoritmos necesarios para realizar operaciones de suma, reta y multiplicación entre expresiones algebraicas adecuadamente. En cuanto a la división, es un proceso que les resulta más complicado a la mayoría ya que no establecen una relación entre la notación como fracción y la operación (simplificación).

Los estudiantes reflejan en sus producciones e intercambios que se han apropiado de 
nuevos conceptos relacionados con la sintaxis algebraica y que han mejorado en los procesos de generalización, no obstante, las falencias que persisten en los pre-saberes aritméticos limitan los avances, toda vez que influyen en los resultados finales.

En relación a la estrategia se concluye que: la dinámica para el desarrollo de las actividades propuesta en la teoría de objetivación: trabajo en grupos reducidos, visita del docente para interacción y retroalimentación, socialización general con aportes entre grupos y retorno al trabajo en grupos (Radford, 2007), fue una alternativa valiosa a integrar en la estrategia didáctica, ya que promueve en los estudiantes la comunicación entre compañeros y con el docente fortaleciendo el proceso de aprendizaje.

Incorporar en el diseño de la estrategia didáctica elementos de la gamificación como la narrativa, los avatares, las recompensas y los puntos, transforma la actitud de los estudiantes frente a la materia, aumentando la participación activa, el compromiso en las diversas actividades y la responsabilidad frente a su proceso de aprendizaje.

La teoría de la objetivación y la gamificación presentan características similares como: el trabajo en equipo, los espacios de intercambio, la autonomía, la retroalimentación constante y la posibilidad de corregir los errores, lo que permite integrarlas en una estrategia didáctica que transforme la dinámica de las clases de matemáticas, siempre realizado con el acompañamiento y evaluación permanente del docente.

\section{RECOMENDACIONES}

Implementar por un espacio de tiempo prolongado estrategias didácticas que integren elementos de la teoría de la objetivación y la gamificación en la enseñanza de las matemáticas en diferentes niveles académicos, teniendo presente la necesidad de acompañamiento $y$, de ser posible, en un ambiente presencial realizar una observación constante sobre los diferentes medios verbales y corporales utilizados en la interacción de los estudiantes. Definir espacios de tiempo de acuerdo a las temáticas o periodos académicos para analizar los resultados obtenidos y la eficiencia de las actividades.

\section{REFERENCIAS BIBLIOGRÁFICAS}

Bonilla-Castro, E., y Rodríguez, P. (1997). Más allá del dilema de los métodos. La investigación en ciencias sociales. Grupo Editorial Norma. https://books.google.com.co/ books?id=REOIWoQuAL4C\&printsec= frontcover\&hl=es\#v=onepage\& $q \& f=f a l s e$

Carr, W. y Kemmis, S. (1988). Teoría crítica de la enseñanza: La investigación acción en la formación del profesorado (J. A. Bravo, Trad.). Ediciones Martínez Roca, S.A. (Original work published 1986).

Chrysostomou, M. y Christou, C. (2019). Analysing the notion of algebraic thinking based on empirical evidence/ Un análisis del concepto de pensamiento algebraico basado en evidencia empírica. Infancia y aprendizaje, 42 (3), 721-781. https:// doi.org/10.1080/02103702.2019.16040 22

Gavilán Bouzas, P. (2011). Dificultades en el paso de la aritmética al álgebra escolar: ¿puede ayudar el Aprendizaje Cooperativo? Investigación En La Escuela, 73, 95-108. https://doi. org/10.12795/IE.2011.i73.07

Hernández, R., Callado, C., y Baptista, M. (2010). Metodología de la investigación, Quinta edición. McGraw-Hill. 
Kapp, K. (2012). The Gamification of Learning and Instruction: Game-based Methods and Strategies for Training and Education. John Wiley \& Sons, Inc.

Kieran, C. y Filloy, E. (1989). El aprendizaje del álgebra escolar desde una perspectiva psicológica. Enseñanza de las ciencias: revista de investigación y experiencias didácticas, 7(3), 229-240.

Martínez-López, L. G., y Gualdron-Pinto, E. (2018). Fortalecimiento del pensamiento variacional a través de una intervención mediada con TIC en estudiantes de grado noveno. Rev. investig. desarro. innov, 9(1), 91-102. doi: 10.19053/20278306. v9.n1.2018.8156

MEN. (2006). Estándares Básicos de Competencias en Lenguaje, Matemáticas, Ciencias y Ciudadanas. https://www.mineducacion.gov.co/1621/
Observatorio de innovación educativa del tecnológico de Monterrey. (2016), Gamificación. EduTrends, 1-36.

Ordóñez-Ortega, O., Gualdrón-Pinto, E., y Amaya-Franky, G. (2019). Pensamiento variacional mediado con baldosas algebraicas y manipuladores virtuales. Rev. investig. desarro. innov., 9 (2), 347-362. doi: 10.19053/20278306. v9.n2.2019.9180

Radford, L. (2014a). De la teoría de la objetivación. Revista Latinoamericana De Etnomatemática Perspectivas Socioculturales De La Educación Matemática, 7(2),132-150.

Radford, L. (2014b). Entrevista con Luis Radford sobre la teoría de la objetivación. Ruta maestra, 9, 33-37.
Radford, L. (2017). Aprendizaje desde la perspectiva de la teoría de la objetivación. En D’Amore, Bruno; Radford, Luis (Eds.), Enseñanza $y$ aprendizaje de las matemáticas: problemas semióticos, epistemológicos y prácticos Énfasis. (pp. 115-136). Bogotá, Colombia: Universidad Distrital Francisco José de Caldas

Vasco, C. (2003). El pensamiento variacional, la modelación y las nuevas tecnologías. [Conferencia]. Congreso Internacional: Tecnologías Computacionales en el Currículo de Matemáticas, Bogotá, Colombia.

Velazco, M. y Mosquera, F. (2007). Estrategias didácticas para el Aprendizaje Colaborativo. https://aprendeenlinea. udea.edu.co/lms/moodle/pluginfile. php/138824/mod_page/content/1/ estrategias_didacticas_aprendizaje_ colaborativo.pdf

Zichermann, G., y Cunningham, C. (2011). Gamification by Design: Implementing Game Mechanics in Web and Mobile Apps. Sebastopol, CA: O’Reilly Media. 